\title{
A importância do diagnóstico precoce da bexiga neurogênica secundária à mielomeningocele na sobrevida renal: relato de caso
}

\author{
The importance of early diagnosis of neurogenic bladder secondary to myelomeningocele \\ in renal survival: case report
}
La importancia del diagnóstico precoz de vejiga neurogénica secundaria a mielomeningocele en la supervivencia renal: relato de caso

Lavínia Ribeiro Tavares $^{1 *}$, Felipe Barros ${ }^{1}$, Igor Barrack Santos ${ }^{2}$, Lara Goulart Pereira ${ }^{1}$, Luiza de Souza Cabral ${ }^{1}$, Luiza Teixeira de Siqueira ${ }^{1}$, Débora da Cruz Cerqueira ${ }^{1}$.

\section{RESUMO}

Objetivo: Descrever um caso clínico e demonstrar as implicações do diagnóstico tardio da Bexiga Neurogênica (BN), consequência da Mielomeningocele (MMC), evidenciando a importância do diagnóstico precoce para prevenção de complicações e melhoria da qualidade e expectativa de vida dos pacientes portadores dessa disfunção. Detalhamento do caso: Paciente do sexo feminino, 42 anos, diagnosticada ao nascimento com MMC. Aos quatro anos, após infecções do trato urinário, obteve diagnóstico de BN e agenesia renal à esquerda, e foi submetida à cistostomia. Assintomática até os 15 anos, manifestou anemia intensa, hiporexia e oligúria, creatinina sérica $13,6 \mathrm{mg} / \mathrm{dL}$ e ureia sérica $177 \mathrm{mg} / \mathrm{dL}$, diagnosticada com doença renal crônica (DRC) e iniciada terapia renal substitutiva. Evoluiu com hipertensão arterial sistêmica, anemia por deficiência de eritropoetina, hiperparatireoidismo secundário com visível déficit de crescimento e hipertrofia importante do ventrículo esquerdo. Considerações finais: Apesar do aumento da expectativa de vida de pacientes com MMC, existem poucos estudos sobre a conduta e o seguimento desses a longo prazo. O desenvolvimento de DRC e as complicações referentes a essa patologia nesses pacientes podem ser evitados. São necessárias mais pesquisas desse tema, visando melhorar a qualidade de vida desses indivíduos e reduzir gastos e comorbidades.

Palavras-chave: Meningomielocele, Bexiga neurogênica, Diagnóstico precoce.

\begin{abstract}
Objective: This article describes a clinical case and demonstrates the implications of the late diagnosis of Neurogenic Bladder (NB), a consequence of Myelomeningocele (MMC), showing the importance of early diagnosis to prevent complications and improve the quality and life expectancy of patients with this dysfunction. Case details: Female patient, 42-year-old, diagnosed at birth with MMC. At the age of four, after urinary tract infections, she was diagnosed with NB and left renal agenesis, and underwent cystostomy. Asymptomatic for upto 15 years, she manifested severe anemia, hypoxia and oliguria, serum creatinine $13.6 \mathrm{mg} / \mathrm{dL}$ and serum urea $177 \mathrm{mg} / \mathrm{dL}$, diagnosed with chronic kidney disease (CKD) and initiated renal replacement therapy. She developed systemic arterial hypertension, erythropoietin deficiency anemia, secondary hyperparathyroidism with visible growth deficit and significant left ventricular hypertrophy. Final considerations: Despite the increase in life expectancy of patients with MMC, there are few studies on their conduct and long-term followup. The development of CKD and complications related to this pathology in these patients can be avoided. More research is needed on this topic, aiming to improve the quality of life of these individuals and reduce expenses and comorbidities.
\end{abstract}

Keywords: Meningomyelocele, Neurogenic bladder, Early diagnosis.

${ }^{1}$ Centro Universitário de Medicina de Belo Horizonte (UNIBH), Belo Horizonte - MG.

${ }^{*}$ E-mail: laviniaribeirot21@gmail.com

2 Faculdade de Medicina do Vale do Aço (UNIVAÇO), Ipatinga - MG.

SUBMETIDO EM: 6/2021

ACEITO EM: 7/2021

PUBLICADO EM: 7/2021 


\section{RESUMEN}

Objetivo: Este artículo describe un caso clínico y demuestra las implicaciones del diagnóstico tardío de Vejiga Neurogénica (VN), consecuencia del Mielomeningocele (MMC), mostrando la importancia del diagnóstico precoz para prevenir complicaciones y mejorar la calidad y esperanza de vida de los pacientes con esta disfunción. Detalles del caso: Paciente de sexo femenino, 42 años, diagnosticada al nacer de MMC. A los cuatro años, después de infecciones del tracto urinario, se le diagnosticó con $\mathrm{VN}$ y agenesia renal izquierda, a la que se le practicó cistostomía. Asintomática hasta los 15 años, manifestó anemia severa, hiporexia y oliguria, creatinina sérica 13,6 mg/dL y urea sérica $177 \mathrm{mg} / \mathrm{dL}$, diagnosticada de enfermedad renal crónica (ERC) e inició terapia renal sustitutiva. Evolucionó con hipertensión arterial sistémica, anemia por deficiencia de eritropoyetina, hiperparatiroidismo secundario con déficit de crecimiento visible e hipertrofia ventricular izquierda significativa. Consideraciones finales: A pesar del aumento de la esperanza de vida de los pacientes con MMC, existen pocos estudios sobre su conducta y seguimiento a largo plazo. Se puede evitar el desarrollo de ERC y las complicaciones relacionadas con esta patología en estos pacientes. Se necesita más investigación sobre este tema, con el objetivo de mejorar la calidad de vida de estos individuos y reducirlos gastos y las comorbilidades.

Palabras clave: Meningomielocele, Vejiga neurogénica, Diagnóstico precoz.

\section{INTRODUÇÃO}

A Mielomeningocele (MMC) ou espinha bífida aberta é uma malformação congênita da coluna vertebral em que ocorre protrusão das meninges, medula espinhal e raízes nervosas. Essa malformação é decorrente da falha de fechamento do tubo neural, evento embrionário que ocorre na quarta semana gestacional em que o ácido fólico é essencial. Assim, a deficiência de folato é o fator de risco mais importante associado à MMC, devendo ser iniciado pelo menos 30 dias antes da fertilização. A doença pode causar repercussões no sistema nervoso, urogenital e músculo-esquelético e consequentemente há considerável morbidade nas crianças sobreviventes (COPP AJ, et al., 2015).

A MMC pode ocorrer em qualquer região da coluna vertebral, sendo mais comum em localização lombosacral, e as lesões produzidas variam de acordo com a localização do defeito e estruturas envolvidas. $O$ acometimento neurológico pode englobar inabilidade de controle vesical e intestinal, hidrocefalia, disfunção cognitiva, deformidades músculo-esqueléticas e alteração de ordem motora ou sensitiva em membros inferiores, acompanhadas ou não de dificuldade ou impedimento para a deambulação (COPP AJ, et al., 2015). O tratamento cirúrgico deve ser realizado de forma precoce, com o objetivo de preservar o máximo possível do tecido nervoso viável (SNOW-LISY DC, et al., 2015).

Apesar da maioria dos portadores de MMC apresentarem função renal inalterada ao nascimento, é fundamental que o paciente seja acompanhado ao longo da vida pelo urologista, pois o acometimento do sistema urinário é uma complicação comum. Uma importante repercussão da $\mathrm{MMC}$ no trato urinário é a Bexiga Neurogênica (BN), definida por disfunção vesico-esfincteriana decorrente de um processo patológico do Sistema Nervoso Central (SNC) ou periférico. Outras causas possíveis de BN são espinha bífida oculta, agenesia sacral, ânus imperfurado, paralisia cerebral, trauma medular e tumores do SNC (STEIN R, et al., 2020). Com o acompanhamento adequado, é possível avaliar a existência da BN, sua extensão e prognóstico, e planejar o prosseguimento, evitando diagnóstico após lesões irreversíveis do trato urinário. $\mathrm{O}$ tratamento deve focar em evitar a má evolução da função vesical e acometimento do trato urinário para prevenir complicações (WIENER JS, et al., 2018).

A BN representa o espectro de distúrbios neurológicos que produz disfunções vesico-esfincterianas em qualquer uma das fases de miç̧ão, seja o armazenamento seguro da urina ou o esvaziamento vesical eficiente, ocasionando incoordenação do funcionamento fisiológico. Dessa forma, a BN pode se manifestar com hipocontratilidade do músculo detrusor da bexiga, em que há incapacidade de esvaziamento eficaz e consequente resíduo urinário pós-miccional; ou com hiperatividade detrusora, que se manifesta por incontinência e urgência miccional. Esses fatores repercutem no trato urinário superior e podem ocasionar refluxo vesico-ureteral (AMARENCO G, et al., 2017). 
Assim, o subdiagnóstico de $\mathrm{BN}$, além de levar à perda da capacidade coordenada de armazenamento e eliminação da urina, resulta em complicações como infecções recorrentes do trato urinário, hidronefrose, nefropatia do refluxo vésico-ureteral, cicatrizes e cálculos renais (AZEVEDO RV, et al., 2014). A ausência de tratamento dessas alterações pode ocasionar lesões e alterações morfológicas no trato urinário superior e inferior, resultando em alguns casos em Doença Renal Crônica (DRC) (AMARENCO G, et al., 2017). Tal condição cursa com diminuição significativa da qualidade de vida decorrente dos distúrbios clínicos e psicológicos que a doença acarreta (IBIAPINA AR, et al., 2016).

A DRC é um problema de saúde pública mundial e sua prevalência pode atingir $50 \%$ em grupos de alto risco, como portadores de BN, sendo mais de três vezes maior que na população geral, apesar dos avanços diagnósticos e terapêuticos (MONTEIRO LM, et al., 2017; SUNG BM, et al., 2018). Embora existam pesquisas acerca da incidência e prevalência de pacientes pediátricos diagnosticados com DRC, poucas analisam a população pediátrica em estágio avançado, já em tratamento substitutivo, principalmente avaliando a qualidade de vida desses pacientes (AZEVEDO TF, et al., 2021).

A apresentação clínica da DRC pediátrica diverge em inúmeras características daquela observada no adulto, sendo possível encontrar, além da queda da taxa de filtração glomerular, comprometimento clínico inespecífico, o que faz com que a DRC seja reconhecida muitas vezes já em estágio avançado. Dentre as modificações encontradas nos pacientes pediátricos, as principais são problemas cardiovasculares, anemia, doença óssea relacionada ao hiperparatireoidismo, anorexia, déficit de crescimento e de desenvolvimento puberal. É válido ressaltar que todos estes possíveis achados descritos na literatura podem ser observados na paciente relatada (BELLORIN-FONT E, et al., 2019; HANNA RM et al., 2020; SOCIEDADE BRASILEIRA DE PEDIATRIA, 2020).

Achados urodinâmicos alterados em crianças são preditivos de deterioração renal e a associação entre encaminhamento precoce para diagnóstico e tratamento especializados e melhoria das alterações já foi comprovada. Nessa lógica, é imprescindível o diagnóstico antecipado da BN em pacientes portadores de MMC com o objetivo de prevenir complicações renais graves e possibilitar melhor qualidade de vida para os pacientes (MONTEIRO LM, et al., 2017; STEIN R, et al., 2020).

Sendo assim, este artigo objetivou relatar um caso clínico e discorrer sobre as implicações do diagnóstico tardio da BN, evidenciando a importância do diagnóstico precoce para prevenção de complicações e melhoria da qualidade e expectativa de vida dos pacientes portadores dessa disfunção.

\section{DETALHAMENTO DO CASO}

As informações clínicas expostas neste relato possuem consentimento da paciente e autorização comprovada por assinatura de Termo de Consentimento Livre e Esclarecido (TCLE). Para sua produção, foram realizados estudo de prontuário médico e entrevista médica. Os dados da primeira infância da paciente, assim como dados do pré-natal materno, são escassos devido à ausência de prontuário médico desta época.

Paciente do sexo feminino, afrodescendente, atualmente com 42 anos, foi diagnosticada ao nascimento com MMC, com correção cirúrgica no período neonatal. Não foi realizado acompanhamento médico durante os primeiros anos de vida da paciente. Aos quatro anos de idade, após episódios de infecção do trato urinário de repetição, foi realizada investigação etiológica, com diagnóstico de BN e agenesia renal à esquerda, sendo optado por cistostomia.

Permaneceu assintomática e sem intercorrências até os 15 anos de idade, quando evoluiu com quadro de anemia intensa, hiporexia e oligúria. Após esse quadro clínico, foram dosados creatinina e ureia séricas, com resultado de 13,6 mg/dL e $177 \mathrm{mg} / \mathrm{dL}$ respectivamente e taxa de filtração glomerular $4 \mathrm{~mL} / \mathrm{min} / 1,73 \mathrm{~m}^{2} \mathrm{com}$ uso da equação Chronic Kidney Disease Epidemiology Collaboration (CKD-EPI). Presença de sinais e sintomas de uremia, histórico de doença do trato urinário inferior e achados laboratoriais contribuíram para o diagnóstico de Doença Renal Crônica agudizada. Foi iniciada terapia renal substitutiva com hemodiálise três vezes na semana, propedêutica mantida até os dias atuais. Ressalta-se que a paciente não é candidata ao transplante renal. 
Paciente evoluiu com hiperparatireoidismo secundário, hipertensão arterial sistêmica e anemia da doença renal crônica por deficiência de eritropoetina. Ecocardiograma realizado aos 37 anos evidenciou hipertrofia importante do ventrículo esquerdo. Com 38 anos de idade foi constatado adenoma de paratireóide, por meio de cintilografia de paratireoides, em topografia de polo inferior direito de glândula tireoide, sendo submetida à paratireoidectomia após alguns meses. Paciente está em uso de losartana $100 \mathrm{mg}$ ao dia, metildopa $500 \mathrm{mg}$ ao dia, eritropoetina $4.000 \mathrm{mg}$ três vezes por semana, suplemento vitamínico do complexo B e ácido fólico, carbonato de cálcio e paricalcitol. Hipertensão não controlada devido ao uso irregular de hipotensores. Atualmente paciente encontra-se em seu $27^{\circ}$ ano de hemodiálise e permanece estável hemodinamicamente, sem intercorrências.

O diagnóstico tardio da BN e a falta de um acompanhamento adequado, principalmente durante a infância, influenciaram negativamente no prognóstico e na qualidade de vida da paciente, com consequências irreversíveis como a doença renal crônica em fase dialítica e múltiplas comorbidades secundárias.

\section{DISCUSSÃO}

Os acometimentos neurológicos associados à MMC são, em sua maioria, permanentes e irreversíveis. A falha no diagnóstico precoce e seguimento de BN cursa com risco aumentado de progressão para degeneração da função renal e comprometimento da morfologia vesical. Sendo assim, o manejo do portador de MMC inclui acompanhamento urológico com objetivo de detectar precocemente possível acometimento do sistema genito-urinário. Por sua vez, pacientes com diagnóstico de BN demandam atenção especial para prevenção de episódios de infecção urinária, cicatrizes renais, má evolução da função vesical e acometimento do trato urinário superior com objetivo de evitar complicações como a DRC (WIENER JS, et al., 2018).

A maioria das crianças portadoras de MMC encontram-se com a função renal inalterada ao nascimento. Portanto, pesquisa urológica complementar deve ser realizada o mais precocemente possível, para avaliar a existência de lesão vesical, assim como sua extensão e prognóstico. A propedêutica da BN é ampla e se baseia em critérios clínicos e exames complementares, o que geralmente inclui ultrassonografia de rins e vias urinárias, uretrocistografia miccional e estudo urodinâmico (AMARENCO G, et al., 2017).

O exame urodinâmico analisa a função do trato urinário inferior, o que permite estratificar o tipo de disfunção miccional e avaliar os principais fatores de risco associados. Com isso, avalia-se a existência de lesão vesical, sua extensão e prognóstico, o que permite planejamento do seguimento do paciente (AMARENCO G, et al., 2017). A International Children Continence Society recomenda a utilização do estudo urodinâmico nos pacientes portadores de MMC que já tiveram a lesão ocluída, como busca ativa da BN (BAUER SB, et al., 2015).

O tratamento precoce tem como objetivo melhorar o prognóstico urodinâmico, reduzindo a gravidade da nefropatia, a incontinência urinária e a infecção do trato urinário, o que provoca significativa melhora na qualidade de vida dos pacientes, mesmo no início da terapia. Com a instituição do tratamento durante o primeiro ano de vida, há possibilidade três vezes maior de melhora urodinâmica desses pacientes (MONTEIRO LM, et al., 2017).

Sendo assim, diante o resultado da urodinâmica, é possível determinar os pacientes que necessitarão ou não de intervenções terapêuticas, que variam desde Cateterismo Intermitente Limpo (CIL) associado ou não a tratamento farmacológico com droga anticolinérgica a derivação urinária externa por vesicostomia ou cistostomia (WIENER JS, et al., 2018). O CIL tem como finalidade o esvaziamento vesical e é fundamental para prevenção de deterioração renal e redução da morbidade. Permite esvaziamento completo da bexiga periodicamente, portanto sua utilização de forma correta impede o aumento da pressão intravesical e 0 acúmulo de resíduo urinário (MAZZO A, et al., 2017; KÓS RS, et al., 2016).

O avanço no tratamento das complicações relacionadas à MMC provocou um aumento da taxa de sobrevivência dos portadores da doença, sendo que, atualmente, um considerável número de crianças chega à fase adulta. Já no século passado até a década de 1950, essa taxa era menor que $10 \%$. Entretanto, os estudos existentes acerca do seguimento desses pacientes e que buscam investigar melhor a população de 
sobreviventes e prevenir possíveis eventos que elevam a morbimortalidade, ainda são escassos e essenciais para propor medidas que objetivam melhorar a qualidade de vida desses indivíduos (ALRIKSSON-SCHMIDT A, et al., 2018; CERVANTE TP, 2017).

A DRC é uma complicação importante da BN que se desenvolve através do refluxo vesicoureteral crônico decorrente do aumento da pressão intravesical. Com isso, os rins sofrem lesão no seu processo morfofuncional, com perda funcional gradativa. Em torno de $40 \%$ das crianças portadoras de BN progridem para a disfunção renal. O comprometimento renal pode ser secundário ao aumento da pressão intravesical, redução da capacidade e complacência da bexiga, dissinergismo vésico-esfincteriano e resíduo pósmiccional, sendo que a identificação destes fatores e a caracterização da disfunção miccional são necessárias para o planejamento do tratamento e prosseguimento do paciente (MONTEIRO LM, et al., 2017).

A BN também predispõe a infecções recorrentes do trato urinário devido à presença de resíduo pósmiccional em consequência do esvaziamento vesical incompleto. Esse processo agrava a lesão da bexiga através da inflamação do parênquima, com consequente substituição por tecido fibrótico e formação de cicatriz renal (WIENER JS, et al., 2018; AZEVEDO RV, et al., 2014). Em relação à paciente do caso clínico, o diagnóstico tardio da bexiga neurogênica associado à falta de seguimento urológico adequado resultou em diagnóstico de lesão renal crônica já em fase terminal, precedido por infecção urinária de repetição.

A população pediátrica portadora de DRC apresenta desafios diferentes em relação às faixas etárias mais avançadas, pois esse grupo está em fase de crescimento e desenvolvimento. Sendo assim, o médico deve se atentar a alguns achados clínicos, laboratoriais e de imagem, como creatinina sérica elevada, taxa de filtração glomerular reduzida, malformação renal bilateral associada, proteinúria persistente, histórico de lesão renal aguda, hipertensão arterial, déficit de crescimento com etiologia desconhecida, noctúria e anemia de difícil manejo. Identificar, prevenir e tratar a DRC e suas complicações na população jovem diminui a provável necessidade de terapia de substituição renal futura (SOCIEDADE BRASILEIRA DE PEDIATRIA, 2020).

A DRC pode ser classificada em cinco estágios a partir dos resultados da taxa de filtração glomerular e da proteinúria do paciente, sendo o estágio cinco caracterizado por lesão irreversível e perda de capacidade renal total, necessitando de terapia de substituição renal com objetivo de remover líquidos e produtos residuais urêmicos do organismo quando o indivíduo não consegue mais fazê-lo (AZEVEDO TF, et al., 2021). A paciente relatada neste artigo está há 27 anos em terapia de substituição renal por hemodiálise, com diagnóstico de DRC estágio cinco desde os 15 anos de idade. Atualmente, milhões de indivíduos no mundo com DRC são tratados por diálise ou transplante (MONTEIRO LM, et al., 2017).

Nesse cenário, é essencial a percepção dos impactos psicossociais causados pelo diagnóstico e tratamento de doenças crônicas na infância ou adolescência. Tendo a DRC em estágio avançado como foco, existem evidências que os pacientes pediátricos com essa comorbidade apresentam pontuações piores de qualidade de vida quando comparados a população saudável de mesma idade (ABRÃO RO, et al., 2021). Considerando pacientes com doença renal crônica em fase terminal, dependentes de diálise, há restrições e mudanças biopsicossociais impostas pelo tratamento, o que interfere diretamente na qualidade de vida (IBIAPINA AR, et al., 2016; SANTOS BP, et al., 2017).

O cotidiano limitado e monótono no tratamento hemodialítico predispõe ao sedentarismo e à deficiência funcional, fatores que refletem significativamente no psicológico e na tendência a desenvolver complicações em outros sistemas orgânicos (SANTOS BP, et al., 2017). Além disso, jovens que passam por intervenções terapêuticas de doenças crônicas muitas vezes apresentam distúrbios psicológicos e comportamentais, como ansiedade, depressão e retraimento social, refletindo diretamente na vida adulta e na qualidade de vida desses indivíduos (ABRÃO RO, et al., 2021). É importante ressaltar que a paciente descrita em caso desenvolveu alterações secundárias à $\mathrm{DRC}$, como déficit de crescimento, que podem influenciar negativamente na qualidade de vida.

Em linhas gerais, sabe-se que a BN é uma importante complicação da MMC que interfere na qualidade e expectativa de vida. O diagnóstico precoce permite acompanhamento urodinâmico e tratamento especializado para evitar ou minimizar repercussões no trato urinário superior. O relato de caso demonstrou portadora de 
MMC com diagnóstico tardio de BN que desenvolveu uma complicação irreversível: lesão renal crônica em fase terminal. Dependência de diálise e alterações secundárias à DRC são fatores que influenciam negativamente a qualidade de vida da paciente. Portanto, é fundamental que os portadores de MMC sejam precocemente encaminhados para acompanhamento com urologista pediátrico. Tal medida pode melhorar a qualidade de vida para a família e o paciente, reduzindo gastos e a morbimortalidade do paciente.

\section{REFERÊNCIAS}

1. ABRÃO RO, et al. Study of the association between generic and disease-specific quality of life and behavior problems in pediatric patients with chronic kidney disease stage 3 or higher and the quality of life and mental health of their primary caregivers. Pediatric Nephrology, 2021; 1-10.

2. ALRIKSSON-SCHMIDT A, et al. Pain and health status in adults with myelomeningocele living in Sweden. Journal Of Pediatric Rehabilitation Medicine, 2018; 11(4): 255-264.

3. AMARENCO G, et al. Diagnosis and clinical evaluation of neurogenic bladder. European Journal Of Physical And Rehabilitation Medicine, 2017; 53(6): 975-980.

4. AZEVEDO RV, et al. Impacto de uma abordagem interdisciplinar em crianças e adolescentes com disfunção do trato urinário inferior (DTUI). Jornal Brasileiro de Nefrologia, 2014; 36(4): 451-459.

5. AZEVEDO TF, et al. Pacientes pediátricos com doença renal crônica em estágio cinco no Brasil: impacto na qualidade de vida. Revista Eletrônica Acervo Saúde, 2021; 13(4): e7294.

6. BAUER SB, et al. International Children's Continence Society standardization report on urodynamic studies of the lower urinary tract in children. Neurourology and Urodynamics, 2015; 34(7): 640-647.

7. BELLORIN-FONT E, et al. Controversies in the Management of Secondary Hyperparathyroidism in Chronic Kidney Disease. Current Osteoporosis Reports, 2019; 17(5): 333-342.

8. CERVANTE TP. Análise dos fatores prognósticos relacionados à mielomeningocele: uma coorte de 20 anos de seguimento. Dissertação (Mestrado em Ciências) - Instituto Nacional de Saúde da Mulher, da Criança e do Adolescente Fernandes Figueira. Fundação Oswaldo Cruz, Rio de Janeiro, 2017; 75 p.

9. SNOW-LISY DC, et al. Update on Urological Management of Spina Bifida from Prenatal Diagnosis to Adulthood. The Journal of urology, 2015; 194(2): 288-296.

10. COPP AJ, et al. Spina bifida. Natural Reviews Disease Primers, 2015; 1(4): 1-18.

11. HANNA RM, et al. Burden of Anemia in Chronic Kidney Disease: beyond erythropoietin. Advances In Therapy, 2020; 38: 52-75.

12. IBIAPINA AR, et al. Aspectos psicossociais do paciente renal crônico em terapia hemodialítica. SANARE, 2016; 15(1): 25-31.

13. KÓS RS, et al. Impacto do tratamento urológico conservador na qualidade de vida de crianças com mielomeningocele e na sobrecarga de seus cuidadores. Estima-Brazilian Journal of Enterostomal Therapy, 2016; 14(2): 84-90.

14. MAZZO A, et al. Qualidade e segurança do cuidado de enfermagem ao paciente usuário de cateterismo urinário intermitente. Escola Anna Nery, 2017; $21(2): 1-7$.

15. MONTEIRO LM, et al. Tratamento precoce melhora o prognóstico urodinâmico na disfunção miccional de origem neurogênica: 20 anos de experiência. Jornal de Pediatria, 2017; 93(4): 420-427.

16. SANTOS BP, et al. Doença renal crônica: relação dos pacientes com a hemodiálise. Arquivos Brasileiros de Ciências da Saúde, 2017; 42(1): 8-14.

17. SOCIEDADE BRASILEIRA DE PEDIATRIA. Doença Renal Crônica em Pediatria: Diagnóstico e Prevenção. Documento Científico n.4, Maio 2020.

18. STEIN R, et al. EAU/ESPU guidelines on the management of neurogenic bladder in children and adolescent part I diagnostics and conservative treatment. Neurology and Urodynamics, 2020; 39(1): 45-57.

19. SUNG BM, et al. Chronic kidney disease in neurogenic bladder. Nephrology, 2018; 23(3): 231-236.

20. WIENER JS, et al. Bladder Management and Continence Outcomes in Adults with Spina Bifida: Results from the National Spina Bifida Patient Registry, 2009 to 2015. The Journal of urology, 2018; 200(1): 187-194. 